\title{
Perda dentária e a imagem do cirurgião-dentista entre um grupo de idosos
}

\section{Tooth loss and the image of the dentist in a group of senior citizens}

Daniel Afonso Hiramatsu ${ }^{1}$

Nilce Emy Tomita ${ }^{1}$

Laércio Joel Franco ${ }^{2}$

'Departamento de

Odontopediatria

Ortodontiaesaúde

Coletiva, Faculdade de

Odontologia deBauru,

Universidade de São Paulo.

Al. Dr Octávio Pinheiro

Brisolla 9-75, Vila

Universitária. 17012-901

Bauru SP.

hiramatsu@usp.br

${ }^{2}$ Departamento deM edicina

Social, Faculdadede

Medicina de Ribeirão Preto,

Universidade deSão Paulo.
Abstract Thisstudy assesses perceptions, memories and beliefs about tooth loss in a group of Japanese-Brazilian senior citizens, selecting forty individuals through a random sampling process, with inclusion criteria related to their background (I ssei/N issei) and oral status (edentulous/nonedentulous), divided into four groups, each with ten subjects. Home interviews were conducted with individuals complying with the inclusion/ exclusion criteria established for each group. U sing the construction of the Collective Subject D iscourse as a methodological strategy, the analysis categories were defined: cause of tooth loss, time of tooth loss and the role of the dentist. Difficulties in accessing dental care and natural acceptance of tooth loss are social and cultural factors that are strongly associated with early edentulism. Pain and fear are the feelings related to the image of the dentist. However, the participants acknowledge that Dentistry has progressed, in terms of equipment, materials and techniques, as well as the training and conduct of dentists. Key words Dentist, Tooth loss, Senior citizens, Qualitative analysis
Resumo 0 presenteestudo busca analisar as percepções, memórias e crenças de um grupo de idosos de origem japonesa quanto às perdas dentárias. Para o presente estudo, foram definidos em processo de amostragem aleatória simples quarenta indivíduos com critérios de inclusão a partir da geração (Issei e Nisei) e condição bucal (edêntulos e dentados), formando quatro grupos com dez indivíduos cada. Foram realizadas entrevistas domiciliares, com os indi víduos que pre encheram os critérios de inclusão-exclusão para cada grupo. Utilizando como estratégia metodológica a construção do Discurso do Sujeito Coletivo, foram definidas as categorias de análise: motivo das perdas dentárias, momento das perdas e o papel do cirurgião-dentista. Dificuldades de acesso à assistência odontológica ea naturalização da perda dentária constituem fatores sociais e culturais fortemente imbricados que resultam em edentulismo precoce. Dor e medo são sentimentos estritamenteligados à imagem do cirurgião-dentista, ainda que se reconheça uma evolução na odontologia, tanto com relação aos equipamentos, materiais e técnicas utilizadas quanto à formação e conduta do profissional. Palavras-chave Cirurgião-dentista, Perda dentária, População idosa, Pesquisa qualitativa 
Introdução

De acordo com o IBGE, a faixa de pessoas com sessenta anos ou mais, em 1960, era responsável por $4,8 \%$ do total da população brasileira. Em 1980, esse número passou para 6,2\% e, em 1999, atingiu $8,7 \%$. M antidas as tendências atuais, a projeção para 2025 é de que a proporção de idosos no país esteja em torno de 15\%. Embora esse percentual seja inferior à média dos países europeus ( $25 \%$ ), coloca para o Brasil a problemática decorrente do envelhecimento, no que diz respeito à saúde (doenças crônicas requerendo cuidados continuados e custosos), agravada pelo fato de que problemas como a desnutrição e doenças infecciosas ainda persistem no país ${ }^{1}$.

No que se refere à saúde bucal, a cárie dentária é uma doença que ainda hoje acomete a população em todos os seus segmentos. Em regiões ou países onde uma proporção significativa da população não tem acesso regular a ações de promoção da saúde bucal e a serviços odontológicos, o tratamento dessa enfermidade, em estágio tardio, é realizado através da extração dos dentes afetados.

Além da cárie, alguns estudos como os de Guimarães \& M $\operatorname{arcos}^{2}$ e M oura et al. ${ }^{3}$ têm mostrado que as doenças periodontais constituem importante causa de exodontias. No Brasil, a perda do órgão dental relacionada a exodontias provocadas por doenças evitáveis, entre elas, a cárie dentária e as doenças periodontais, é muito elevada. Dados epidemiológicos têm mostrado expressivo incremento das perdas com a idade. Em 1986, estimava-se que $10 \%$ da população brasileira aos 34 anos de idade apresentava ausência total de dentes. Aos 41 e 48 anos de idade, esse problema atingia, respectivamente, 20 e $30 \%$ dos brasileiros. A partir dessa idade, a proporção de edêntulos é cada vez maior e o colapso da dentição é mais intenso: $40 \%$ aos 53 anos, 50\% aos 58 anos; $60 \%$ aos 63 anos; $70 \%$ aos 68 anose $80 \%$ aos 70 anos de idade.

Um dos principais indicadores de risco para o edentulismo é a perda dentária precoce4. Informação epidemiológica sobrea perda dentária precoce em adultos brasileiros foi obtida para o grupo etário de 35 a 44 anos de idade em levantamento epidemiológico realizado em 1986 em dezesseis capitais. N essa faixa etária, a prevalência de cárieera de 22,5 dentes atacados, caracterizando um valor "muito alto", segundo a classificação de prevalência apresentada por M urray ${ }^{5}$. De cada três dentes atacados por cárie, dois haviam sido extraídos, correspondendo a uma média de cerca de quinze dentes perdidos por adulto.

Através da história, observa-se que, inicialmente, a prática odontológica era primitiva e rudimentar. Em algumas sociedades, tais práticas eram usadas, inclusive, como forma de penalidade etortura a quem transgredisse as leis $s^{6,7}$. A pesar de se reconhecer um progresso importante nos tratamentos odontológicos, a literatura leiga afirma queuma das características da ciência quecuida da saúde da boca é evoluir muito lentamente e que "dentista sem dor é coisa do futuro".

$\mathrm{N}$ a literatura romanesca, percebemos que ele é retratado, em geral, de forma negativa, aparecendo como uma pessoa má e ridícula. Essa imagem é, também, veiculada pela TV e cinema $8,9,10,11,12,13$. Freqüentemente, o papel do cirurgiãodentista é associado a práticas odontológicas mutiladoras representadas pelas exodontias. Conseqüentemente, as pessoas trazem consigo uma carga de medo e ansi edade muito grandeao chegar ao consultório odontológico.

Essa mentalidade contribui para que, atualmente, no Brasil, a saúde bucal venha sendo relegada ao esquecimento quando se discutem as condições de saúde da população idosa. Considerando que essas concepções de saúde e doença estão relacionadas às raízes das pessoas, sendo influenciadas por suas experiências, suas concepções se constroem em relação ao seu lugar e posição na sociedade ${ }^{14}$. Não fugindo a essa regra, as representações de saúde bucal são construídas através da vivência do cotidiano e de reinterpretações do discurso do profissional. Essas imagens surgem como reflexo das realidades objetivas de cada indivíduo, determinadas histórica e culturalmente ${ }^{15}$.

Assim, a perdatotal dedentes (edentulismo), ainda éaceita pela sociedade como al go normal e natural com o avanço da idade, e não como re flexo da falta de políticas públicas no campo da saúde bucal, destinadas principalmente à população adulta, para que mantenha seus dentes até idades mais avançadas ${ }^{16,17}$.

Visando verificar, na população idosa brasileira, de origem japonesa, as causas da perda dentária, buscou-se analisar sua influência na percepção da odontologia e do cirurgião-dentista no processo saúde-doença-cuidado que resulta em mutilação. 
Método

O protocolo de pesquisa foi aprovado pelo ComitêdeÉtica em Pesquisa da Faculdade deO dontologia de Bauru-USP, atendendo à Resolução 196/96 do Conselho Nacional de Saúde.

Este trabalho apresenta um caráter qual itativo. Na pesquisa qualitativa, éo conjunto de situações que dá sentido, e não uma situação isolada. Pode-se, entretanto, buscar o importante na novidade dos temas mesmo se a freqüência for pequena ${ }^{18}$.

Visando um estudo em profundidade do tema, considerou-se, portanto, que uma análise qualitativa seria o melhor método para fornecer as categorias necessárias para uma comprovação posterior através de uma quantificação rigorosa.

A população estudada foi composta de quarenta indivíduos nipo-brasileiros, da primeira e segunda gerações, na faixa etária de 60 a 80 anos, de ambos os sexos, residentes no município de Bauru-SP.

Para a identificação da população-alvo, foi realizado um censo, em 1998, sob coordenação do Departamento de M edicina Preventiva da Universidade Federal de São Paulo (UNIFESP), com a colaboração do Clube Cultural N ipo-Brasileiro de Bauru. Foi cadastrada a totalidade de indivíduos nipo-brasileiros da primeira geração (Issei) e da segunda geração (Nisei), com idade acima de 30 anos, de ambos os sexos, residentes no município de Bauru, resultando em 1.773 indivíduos, que foram convidados a participar de estudos sobre a saúde da população nikkei ${ }^{19}$.

Para o presente estudo, foram definidos em processo de amostragem aleatória simples quarenta indivíduos com critérios de inclusão a partir da geração (Issei e Nisei) e condição bucal (edêntulos e dentados), formando quatro grupos, com dez indivíduos cada. Foram realizadas, por entrevistador único, as entrevistas domiciliares com os indivíduos que preencheram os critérios de inclusão-exclusão para cada grupo ( Tabela 1). Estas entrevistas foram gravadas e posteriormente transcritas.

U tilizou-se como estratégia metodológica em pesquisa qualitativa a construção do Discurso do Sujeito Coletivo ${ }^{20}$, que consiste numa forma qualitativa de representar o pensamento de uma coletividade, agregando em um discurso-síntese os conteúdos discursivos semelhantes emitidos por pessoas distintas. Cada indivíduo entrevistado no estudo, escolhido com base em critérios de representatividade social, contribui com sua cota de fragmento de pensamento para o pensamento coletivo ${ }^{20}$.

Este procedimento metodológico pressupõe a definição, a partir deuma perspectiva empírica, dequeo caráter coletivo do pensamento social éa quantidade de escolhas de um determinado conjunto de indivíduos pertencentes a uma determinada coletividade e, apesar de expresso de forma individualizada, ésocialmentecompartilhado, traduzindo a natureza do pensamento coletivo ${ }^{20}$.

Fez parte do processo de coleta de informações a entrevista utilizando roteiro semi-estruturado. O ptou-se pelo roteiro aberto para recoIher o maior número possível de percepções, captando a visão própria dos indivíduos. As categorias de análise estabelecidas foram: motivo das perdas dentárias, quando ocorreram as perdas e o papel do cirurgião-dentista.

\section{Discussão}

Com relação às categorias estudadas, os achados guardam grande semelhança independente de sexo, idade, geração (I ssei ou $\mathrm{N}$ isei) ou condição bucal (edêntulo total ou parcial).

Tabela 1. M édia etária em anos dos entrevistados segundo a geração e edentulismo. Bauru-SP, 2004.

\begin{tabular}{|c|c|c|c|c|c|c|c|c|}
\hline & \multicolumn{4}{|c|}{ ISSEI } & \multicolumn{4}{|c|}{ NISEI } \\
\hline & \multicolumn{2}{|c|}{ masculino } & \multicolumn{2}{|c|}{ feminino } & \multicolumn{2}{|c|}{ masculino } & \multicolumn{2}{|c|}{ feminino } \\
\hline & média & (n) & média & (n) & média & $(n)$ & média & (n) \\
\hline Com dentes & 65,5 & (7) & 63 & (3) & 59,6 & (3) & 56 & (7) \\
\hline Edêntulos & 71,5 & $(2)$ & 67 & (8) & 60 & $(2)$ & 66,7 & (8) \\
\hline
\end{tabular}


Guardando a confidencialidade, a transcrição de algumas expressões é feita a seguir, com objetivo de subsidiar a discussão, utilizando-se números dispostos aleatoriamente para referenciar as falas dos diferentes sujeitos.

a) Motivo das perdas

Deu muita dor de dente. Sofri viu, eu fiquei nervosa, mandei arrancar para livrar da dor de dente. Foi caindo assim, aí precisou arrancar tudo. Para tratar dava trabalho, tinha cárie nos dentes.

A cárie foi citada, pela maioria dos entrevistados, como principal fator etiológico das perdas dentárias.

Porquemorava no sítio, enão tratava dos dentes, tava cariando muito, deixava estragar[...]

Percebe se que 0 acesso ao tratamento odontológico era dificultado seja pela escassez de profissionais ou pelo próprio impedimento físico, já que a maioria da população estudada morava na zona rural, o que permitia a evolução da cárie que freqüentemente levava a exodontias múltiplas para posterior instalação de próteses totais como forma de tratamento disponível à época.

Porque tava tudo estragado, não tratava dos dentes, não tinha dinheiro pra tratar. Eu sempre senti dor, já não dava mais, tinha ponte móvel, pivot, obturação, caiu tudo .

[...] mas no meu tempo era difícil, na minha cidade, por exemplo, tinha um dentista só e era caríssimo.

0 alto custo dos tratamentos e a precária situação financeira dos entrevistados na época da perda dos dentes foram citados como fatores limitantes para 0 acesso ao tratamento odontológico.

\section{b) Quando ocorreram as perdas}

Já faz uns 44 anos. Eu era solteira ainda, acho que eu tava com 20 anos.

Nota-se que os indivíduos edêntulos da população estudada passaram a essa condição por volta dos 20 a 30 anos de idade. 0 que se pode notar é que esses indivíduos perderam os dentes em uma faixa etária muito baixa, o que nos dá idéia das dificuldades de acesso a informações e tratamento quanto à saúde bucal pelas quais passaram.

Foi na gravidez da minha menina, eu quase morri de dor de dente, aí que eu ganhei a menina, passou uns dois meses eu mandei tirar tudo. $M$ as eu fiquei nervosa! Fiquei tudo, eu não dormia, ah eu sofri. Aí eu fiquei nervosa, fui no dentista, mandei tirar tudo, em cima e embaixo.

Aqui, o mito das perdas dentárias durante a gestação pode ser confirmado, já que este período foi citado como crítico para a saúde bucal, em que grandes perdas dentárias ocorreram.

Isso aí quando eu tinha uns 25 a 30 anos eu acho [...] muito nova, foi depois que casei.

Eu era solteira ainda, acho que eu tava com 20 anos, tirei tudo junto, nem precisava tirar tudo, mas tirei.

N este caso, muitos utilizaram como pontos de referência eventos marcantes da vida (casamento, gravidez, nascimento dos filhos) como recurso para auxiliar na determinação da idade aproximada em que ocorreu o evento "perda dos dentes".

\section{c) 0 papel do cirurgião-dentista}

Pôde-se perceber que o próprio cirurgiãodentista sugeria que fosse feita a exodontia total, o que revela as práticas odontológi cas mutiladoras, sendo que esse procedimento fazia parte da conduta profissional naquele contexto histórico como forma de tratamento largamente utilizada. Os entrevistados referem que os profissionaisressal tavam a condição de edentulismo como uma forma prática, definitiva e de baixo custo para solucionar os problemas de saúde bucal.

Acho que o dentista falou para o meu pai, arranca tudo, põe dentadura quenunca mais dá dor de dente, não precisa o senhor ficar trazendo para tratar. Naquela época, qualquer coisinha já falava para tirar, não tentava salvar.

Pode-se observar que as extrações dentárias em série constituíam parte da cultura desses indivíduos quanto à saúde bucal. A perda dos dentes era considerada uma etapa da evolução da condição bucal do indivíduo, ou seja, o edentulismo era tido como uma condição normal einevitável com o decorrer da idade. Em muitos casos, a decisão de extrair todos os dentes foi do próprio paciente.

Decisão minha, porque fica sofrendo de dor. Eu pensava assim: eu não vou esperar dar dor, eu vou arrancar antes de dar dor. Eu falei que queria tirar tudo, tava sempre doendo.

[ ...] os pais não se preocupavam tanto assim, então eu perdi (os dentes) jovem.

A associação da imagem do cirurgião-dentista à dor aparece nos relatos de forma consistente. A dor não vem associada a qualquer estímulo específico de forma marcante, apenas ao tratamento e ao dentista de uma forma geral. A 
associação da dor a estímulos específicos, quando ocorre, envolveinstrumentais etraduz, decerta forma, situações de agressão à integridade do paciente, como uso de broca/motor e agulha/ anestesia.

M as tinha uns dentes bons ainda, eu tinha medo de ir ao dentista, daquela injeção [...] eu chorava.

la obturar dente, naquele tempo punha máquina, faz aquele barulho sabe?

0 medo está presente, na grande maioria das entrevistas, fortemente associado à imagem do dentista. Esse sentimento faz parte do cotidiano da relação dentista-paciente, aparecendo nos relatos tanto como uma sensação do entrevistado, como das pessoas as quais ele convive. Observase, dessa forma, que o medo é comum, natural e inquestionado.

Com relação aos estímulos específicos causadores de medo, os entrevistados citam algumas vezes o instrumental e o tratamento em si. Assim como nos trabal hos deK leinknecht et al. ${ }^{21}$, Rankin \& $\mathrm{Harris}^{22}$ eO liveira ${ }^{23}$, o medo éassociado, principalmente, à agulha e ao motor.

Já estava tudo estragado e para tratar ficava aquele preço, então falei, arranca tudo evamos pôr dentadura. Como eu não tinha condições de pagar, podia arrancar, eu falei.

Alguns relatos mostram uma certa angústia, diante dos altos custos do tratamento odontológico. Liu²4 relata que a inconsciência social da Odontologia tem exercido um grande papel no declínio da confiança do público.

De maneira geral, os entrevistados reconhecem os avanços alcançados pela O dontologia. Ressaltam a evolução das técnicas, dos materiais e da própria formação do cirurgião-dentista.

Ah melhor, mil vezes melhor. Eu acho que agora o dentista tem bem mais informação, bem mais conhecimento do que antigamente.

A hojemudou tudo, como a medicina, a odontologia, tudo, tudo, a evolução émuito grande, não dá nem pra comparar. Hoje as técnicas estão mais avançadas, tem aparelhamento, o pessoal está mais orientado, orientam mais. Antigamente o dentista mesmo que falava que era melhor arrancar. $M$ as agora não, trata até o fim.

A gora émelhor [...] está bem adiantado agora, viu. Os materiais, tratamento, tudo, não dá para comparar com 0 antigo.

São observados relatos evidenciando o profissional dos dias atuais como al guém que esclarece e orienta seus pacientes, utilizando métodos mais conservadores do que no passado.
H oje um dentista orienta o cliente [...] antes não. Sefosse hoje, duvido queo dentista mearrancaria o dente, ele iria procurar fazer tratamento.

Hoje em dia o dentista explica melhor.

H oje está melhor, na época [...] o dentista não dava essa orientação de escovação, nesse sentido a importância do dentista aumentou sim, bastante [...] hoje em dia, eles vêem toda parte da boca. Se fosse agora, jamais teria deixado tirar os dentes. Hoje, quando dá pra tratar, ele trata, não deixa tirar.

A pesar deem número reduzido, alguns entrevistados relataram não encontrar diferenças entre a Odontologia praticada no passado quando comparada à dos dias atuais. Percebe-se que, nesses casos, essa opinião pode expressar alguma falta deconhecimento sobrea odontologia advinda das dificuldades de acesso ao cirurgião-dentista.

Acho que é a mesma coisa, pelo menos para mim acho quenão deu diferença, porque o método detratamento, acho quenão mudou, sempreigual.

\section{Consideraçõesfinais}

A dificuldade de acesso ao tratamento odontológico, pela escassez de profissionais na época ou pelo fato de que muitos viviam na zona rural, o alto custo dos tratamentos ou a própria mentalidade, tanto da população quanto dos profissionais, foram fatores que contribuíram para que as perdas ocorressem em idades tão precoces, resultando em elevado edentulismo.

Os discursos referem que o cirurgião-dentista desempenhou papel importante na perda precoce de dentes, uma vez que em muitos casos a decisão pelas exodontias foi fortemente influenciada pelo profissional.

0 elevado custo do tratamento odontológico esteve presentenos relatos como um fator que impossibilitou tratamentos mais adequados, contribuindo para a perda precoce de dentes.

Dor e medo são sentimentos estritamente ligados à imagem do cirurgião-dentista, ainda que se reconheça uma evolução na odontologia, tanto com relação aos equipamentos, materiais e técnicas utilizadas, quanto à formação econduta do profissional.

\section{Agradecimentos}

Agradecemos o apoio da Fundação de Amparo à Pesquisa do Estado de São Paulo (FAPESP). 


\section{Colaboradores}

DA H iramatsu realizou as entrevistas, a transcrição das respostas e a discussão dos achados, NE Tomita trabalhou na concepção e redação e LJ Franco participou da discussão.

\section{Referências}

1. Ramos LR, Veras RP, Kalache A. Envelhecimento populacional: U ma realidade brasileira. Rev Saúde Pública 1987; 21:211-224.

2. Guimarães $M M, M$ arcos B. Perda de dente relacionada a razões clínicas segundo a classe social. Rev CROM G 1995; 1(2):54-61.

3. Moura WL, Eugênio MJE, Silva EF. Causas determinantes de exodontias na clínica cirúrgica do curso de odontologia da Universidade Federal do Piauí. Rev Assoc Saúde Pub Piauí 1998; 1(1):71-83.

4. Eklund S A, Burt B A. Risk factors for tooth loss in the United States: Iongitudinal analysis of national data. J Public Health Dent 1994; 54:5-14.

5. Murray JJ. Appropriate use of fluorides for human health. Geneva: World Health Organization; 1986.

6. Guerini V. History of dentistry: from the most ancient times until the end of eighteenth century. Part I, first period - antiquity - introduction. New York: Lea \& Febiger; 1909. p. 17-18.

7. Resende VLS. A história da Odontologia. Belo Horizonte: Faculdade de Odontologia da UFM G; 1994.

8. Martinez $\mathrm{H}$. Interpreters of mumblings. $\mathrm{Br}$ Dent J 1990; 169(11):348

9. Pride J. Dealing with dentistry's dilemma. J Am Dent Assoc 1991; 122(10):91-92.

10. Gerbert B. How dentists see themselves, their profession, the public. J Am Dent Assoc 1992; 123(12):7278.

11. M eskin LH. A matter of trust. J Am Dent Assoc 1992; 123(7):8-11.

12. Schuman NJ. Dentistry as portrayed in motion pictures on television. Compend Contin Educ Dent 1993; 14(1):102-106.

13. Gerbert B. Dentists and patients who love them: professional and patients view of dentistry. J Am Dent Assoc 1994; 125(3):265-272.

14. Minayo M CS. Na dor do corpo, o grito da vida. In: Costa $\mathrm{N}$ do $\mathrm{R}$, et al., organizadores. Demandas populares, políticas públicas e saúde. Petrópolis: Editora Vozes; 1989. p.75-99.
15. Bernd B, et al. Percepç̃o popular sobre saúde bucal: o caso das gestantes do Valão. Saúde em Debate 1992; 34:33-39.

16. Pucca Jr. GA. A saúde bucal do idoso? Aspectos demográficos e epidemiológicos. M edcenter 2000. [acessado 2005 Set 15]. Disponível em: http:// www.odontologia.com.br/

17. Rosa AGF, Fernandez RAC, Pinto VG, Ramos LR. Condições de saúde bucal em pessoas de 60 anos ou mais no Município de São Paulo (Brasil). Rev Saúde Pública 1992; 26:155-160.

18. Minayo M CS. 0 desafio do conhecimento: pesquisa qualitativa em saúde. São Paulo: Hucitec; Rio de Janeiro: Abrasco; 1993.

19. Tomita NE, Chinellato LEM, Pernambuco RA, Lauris JRP, Franco LJ. Condições periodontais e diabetes mellitus na população nipo-brasileira. Rev Saúde Pública 2002; 36(5):607-613.

20. Lefèvre $F$, Lefèvre AM C. 0 discurso do sujeito coletivo: um novo enfoque em pesquisa qualitativa (desdobramentos). Caxias do Sul: Ed. Educs; 2003.

21. Kleinknecht RA. Origins and characteristics of fear of dentistry. J Am Dent 1973; 86(4):842-848.

22. Rankin JA, Harris M B. Dental anxiety: the patient's point of view. J Am Dent Assoc 1984; 109(1):43-47.

23. Oliveira KM L. M edo, ansiedade e a prática odontológica. Semana de Iniciação Científica da UFM G. Belo Horizonte; 1993.

24. Liu JR. Social issues. J Am Dent Assoc 1992; 123(11):18-19

Artigo apresentado em 18/10/2005

Aprovado em 15/03/2006

Versão final apresentada em 24/11/2006 